\title{
The Interplanetary Network Database
}

\author{
K. Hurley ${ }^{1}$ \\ U.C. Berkeley Space Sciences Laboratory \\ 7 Gauss Way, Berkeley, CA 94720-7450, U.S.A. \\ E-mail: khurley@ssl.berkeley.edu
}

\section{G. Mitrofanov, D. Golovin, M. L. Litvak, A. B. Sanin}

Institute for Space Research

Profsojuznaja 84/32, Moscow 117997, Russian Federation

E-mail: imitrofa@space.ru

W. Boynton, C. Fellows, K. Harshman, R. Starr

University of Arizona, Lunar and Planetary Laboratory

Tucson, AZ 85721, U.S.A.

E-mail:wboynton@gamma1.lpl.arizona.edu

\section{S. Golenetskii, R. Aptekar, E. Mazets, V. Pal'shin, D. Frederiks, D. Svinkin}

Ioffe Physico-Technical Institute of the Russian Academy of Sciences

St. Petersburg, 194021, Russian Federation

E-mail: golen@mail.ioffe.ru

\section{M. Smith}

Department of Physics and Santa Cruz Institute for Particle Physics University of California, Santa Cruz 95064 CA, U.S.A.

E-mail:dsmith@scipp.ucsc.edu

\section{W. Hajdas}

Paul Scherrer Institute

5232 Villigen PSI, Switzerland

E-mail:wojtek.hajdas@psi.ch

\section{A. von Kienlin, X. Zhang, A. Rau}

Max-Planck-Institut für extraterrestrische Physik

Giessenbachstrasse, Garching, 85748 Germany

E-mail: azk@mpe.mpg.de

\section{K. Yamaoka}

Department of Physics and Mathematics, Aoyama Gakuin University

5-10-1 Fuchinobe, Sagamihara, Kanagawa 229-8558, Japan

E-mail: yamaoka@phys.aoyama.ac.jp

$1 \quad$ Speaker 


\section{T. Takahashi}

Institute of Space and Astronautical Science (ISAS/JAXA)

3-1-1 Yoshinodai, Sagamihara, Kanagawa 229-8510, Japan

E-mail: takahasi@astro.isas.ac.jp

\section{Ohno, Y. Hanabata, Y. Fukazawa}

Department of Physics, Hiroshima University 1-3-1 Kagamiyama, Higashi-Hiroshima, Hiroshima 739-8526, Japan

E-mail: ohno@hep01.hepl.hiroshima-u.ac.jp

\section{Tashiro,Y. Terada}

Department of Physics, Saitama University

255 Shimo-Okubo, Sakura-ku, Saitama-shi, Saitama 338-8570, Japan

E-mail: tashiro@phy.saitama-u.ac.jp

\section{T. Murakami}

Department of Physics, Kanazawa University

Kadoma-cho, Kanazawa, Ishikawa 920-1192, Japan

E-mail:murakami@astro.s.kanazawa-u.ac.jp

\section{K. Makishima ${ }^{2}$}

Department of Physics, University of Tokyo

7-3-1 Hongo, Bunkyo-ku, Tokyo 113-0033, Japan

E-mail:maxima@phys.s.u-tokyo.ac.jp

\section{T. Cline ${ }^{3}$, S. Barthelmy, J. Cummings ${ }^{4}$, N. Gehrels, H. Krimm ${ }^{5}$}

NASA Goddard Space Flight Center

Code 661, Greenbelt, MD 20771, U.S.A.

E-mail: Thomas.L.Cline@nasa.gov

\section{Palmer}

Los Alamos National Laboratory

P.O. Box 1663, Los Alamos, NM 87545, U.S.A.

E-mail: palmer@lanl.gov

\section{J. Goldsten}

Applied Physics Laboratory, Johns Hopkins University

Laurel, MD 20723, U.S.A.

E-mail: john.goldsten@jhuapl.edu

\section{E. Del Monte, M. Feroci}

INAF/IASF-Roma

\footnotetext{
${ }^{2}$ Also Makishima Cosmic Radiation Laboratory, The Institute of Physical and Chemical Research (RIKEN), 2-1 Hirosawa, Wako, Saitama 351-0198, Japan

${ }^{3}$ Emeritus

${ }^{4}$ Also Joint Center for Astrophysics, University of Maryland, Baltimore County, 1000 Hilltop Circle, Baltimore, MD 21250

${ }^{5}$ Also Universities Space Research Association, 10211 Wincopin Circle, Suite 500, Columbia, MD 21044
} 
via Fosso del Cavaliere 100, 00133, Roma, Italy

E-mail:sa.grb@iasf-roma.inaf.it

\section{Marisaldi}

INAF/IASF-Bologna

Via Gobetti 101, I-40129 Bologna, Italy

E-mail: marisaldi@iasfbo.inaf.it

\section{Connaughton, M. S. Briggs}

University of Alabama in Huntsville

NSSTC, 320 Sparkman Drive, Huntsville, AL 35805, U.S.A

E-mail: connauv@uah.edu

\section{Meegan}

Universities Space Research Association

NSSTC, 320 Sparkman Drive, Huntsville, AL 35805, U.S.A.

E-mail: charles.a.meegan@nasa.gov

Interplanetary Networks (IPNs) have been in use since the late 1970's. The current IPN comprises 9 spacecraft and detects about $325 \mathrm{GRBs} /$ year, roughly 100 of which are not localized by Swift or Fermi. It is an all-sky, full-time monitor for short-duration gamma-ray transients. We describe the IPN database, which is publicly available on the IPN website. It comprises, among other things, a GRB bibliography, a comprehensive list of bursts from 1990 to the present, and localization data. We also describe some of the uses of the IPN data. 


\section{Introduction}

In the beginning (ca. 1975 A.D.), the only way to get arcminute GRB positions was by triangulation. Consequently, the IPN has a long history, and over 30 spacecraft have participated in it (figure 1). But it also has a present and a future, and that is what will be emphasized in this paper.

IPN MISSION TIMELINES, 1976 - PRESENT

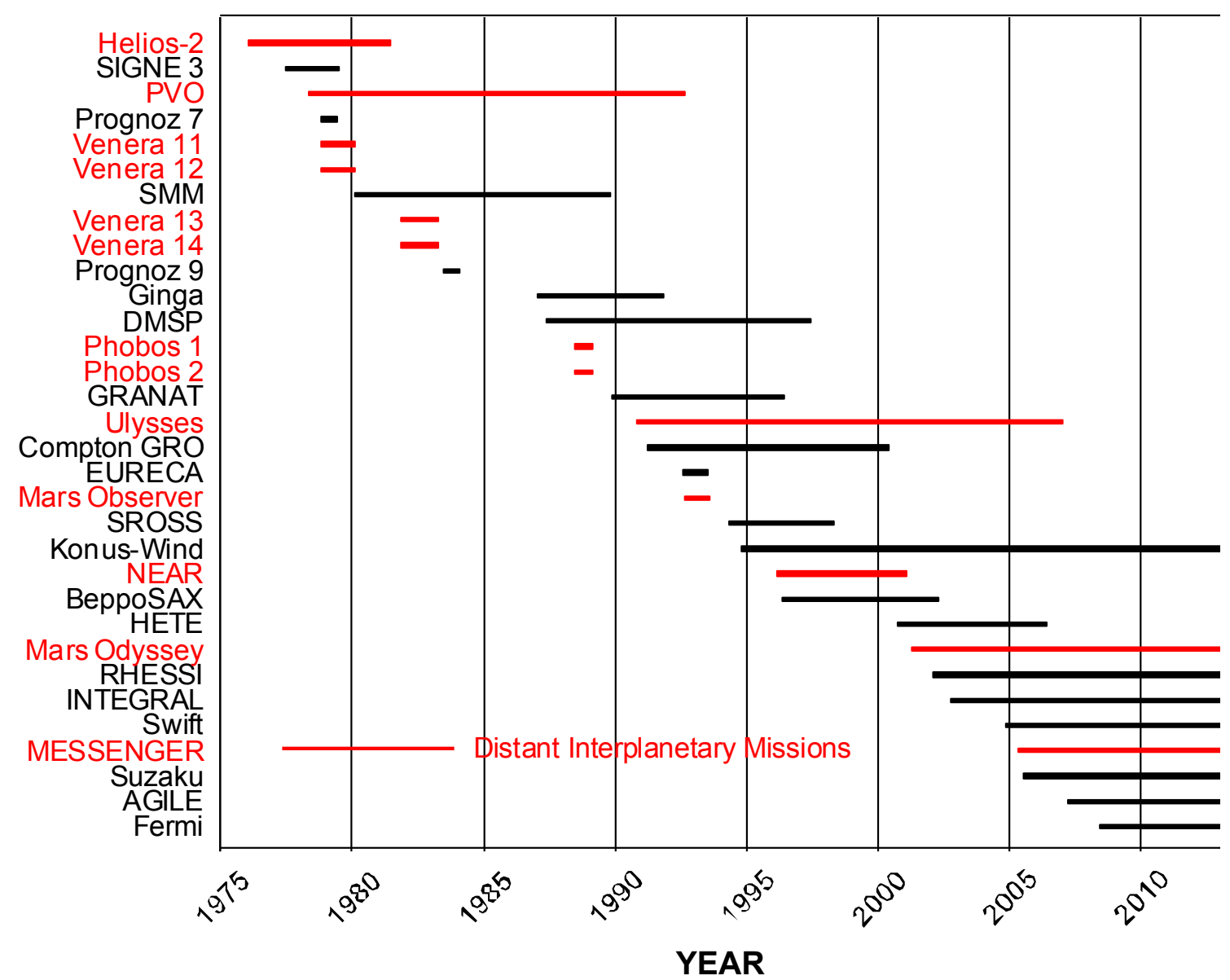

Figure 1. The timelines of some of the missions which have participated in the interplanetary network since its inception. The red lines indicate the distant interplanetary missions, while the black lines are for the missions which were closer to Earth. 


\subsection{The Current IPN}

Presently, the IPN comprises 9 spacecraft. They are AGILE, Fermi, RHESSI, Suzaku, and Swift, in low Earth orbit; INTEGRAL, in an eccentric orbit with apogee 0.5 light-seconds; Wind, at 6 light-seconds from Earth; MESSENGER, in orbit around Mercury, at a distance of roughly 300 to 700 light-seconds; and Odyssey, in orbit around Mars, at a distance of roughly 260 to 1260 light-seconds. It is one of the best configurations that the IPN has ever had, since it has not only two distant spacecraft, but also, a mini-network closer to Earth.

In this configuration, the IPN detects about $325 \mathrm{GRBs} / \mathrm{year}$ and it effectively acts as a fulltime, all-sky monitor for gamma-ray transients (mainly SGRs and GRBs). "Full time" means that there is no time when all the spacecraft are off. "All-sky" means that there is no point in the sky which is ever invisible to all the missions. Indeed, 3 of them (INTEGRAL, MESSENGER, and Wind) have essentially no planet-blocking. The Venn diagram of figure 2 shows the relation between the bursts detected by Swift, Fermi, and the IPN.

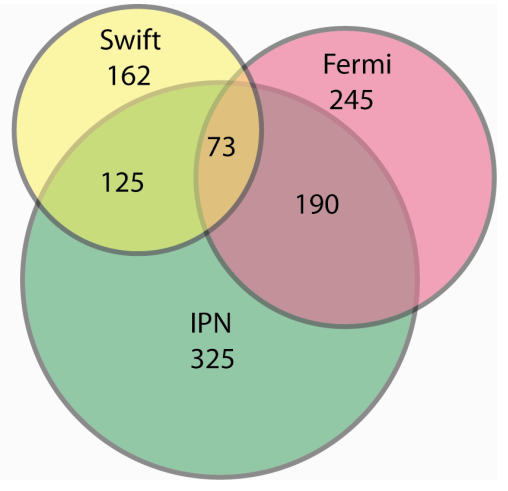

Figure 2. The relation between the GRBs observed by Swift, Fermi, and the IPN. The yearly rates are shown. The Swift bursts are those both within and outside the coded field of view of the BAT (the latter rate slightly exceeds the former). The IPN detects about 100 bursts per year which are not observed by either Fermi or Swift.

The sensitivity of the IPN is primarily a function of the GRB fluence and peak flux. To a much lesser extent, it also depends on the burst duration and other factors. Integrating over peak fluxes and durations, we can derive the sensitivity as a function of fluence. This is shown in figure 3.

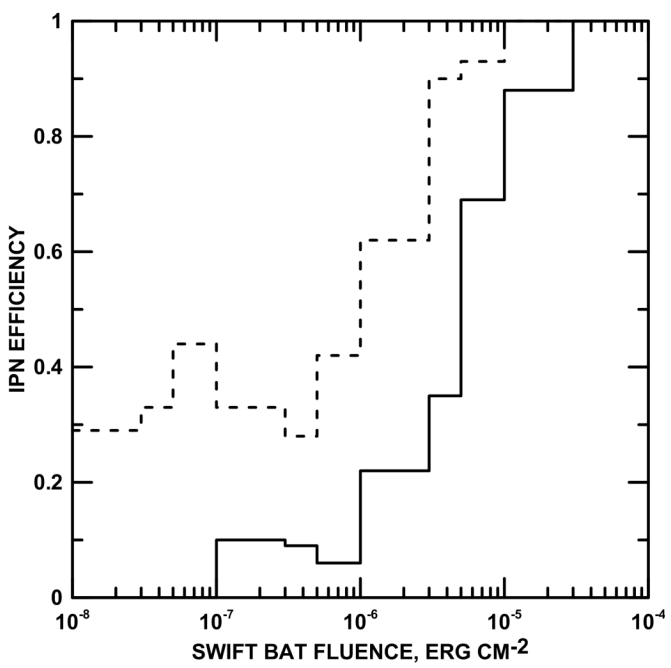

Figure 3. The IPN sensitivity as a function of fluence. The sensitivity is expressed here as the efficiency, or probability of detecting a burst; the fluence is that measured by the Swift BAT in the $15-150 \mathrm{keV}$ range. The dashed line shows the probability that any two IPN spacecraft will detect a burst; if they are both near-Earth spacecraft, this will not result in a triangulation because the separation is too small. The solid line shows the probability that two or more widely separated spacecraft will detect a burst; these observations can be used to triangulate the event. 


\section{The Database}

The IPN database (ssl.berkeley.edu/ipn3/index.html) has three main sections. The first is a GRB bibliography with over 11,000 titles published between 1972 and the present. The second is a table of 25,000 events, mostly GRBs, SGRs, and solar flares, which occurred between 1990 and the present. The table contains their dates and times, and indicates which spacecraft observed them. The third is a table of about 7000 GRB localizations from 1990 to the present. These numbers are increasing steadily with time. The first and third sections are updated semiautomatically on a roughly daily basis, while the second is updated on a roughly monthly basis.

The localizations are expressed as small circles on the celestial sphere. They may intersect to form neat, small polygons (figure 4), or large, irregular areas (figure 5). The user does not need to be concerned with error box shapes, however, because programs are available to answer the most frequently asked questions, namely, "did any GRBs occur during a given time period which had positions consistent with a given RA and Dec, and if so, what were the areas of their error boxes?"

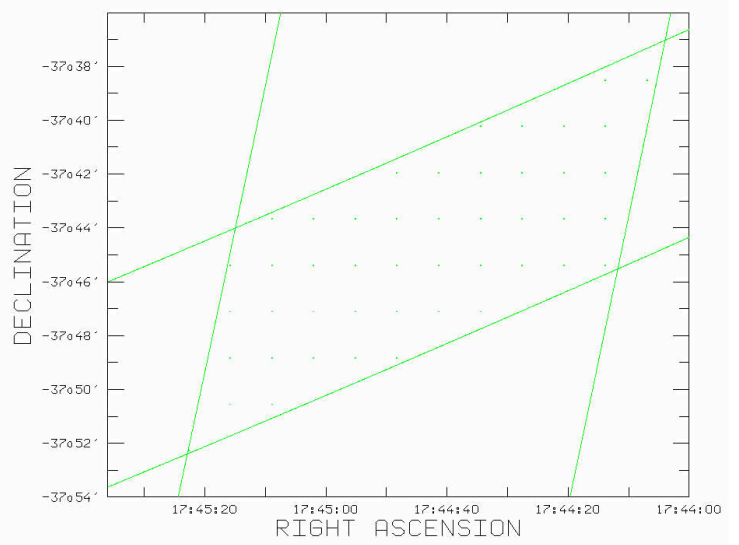

Figure 4. A small error box for GRB 110808 , formed by the intersection of two IPN annuli. The region shaded in green defines the error box.

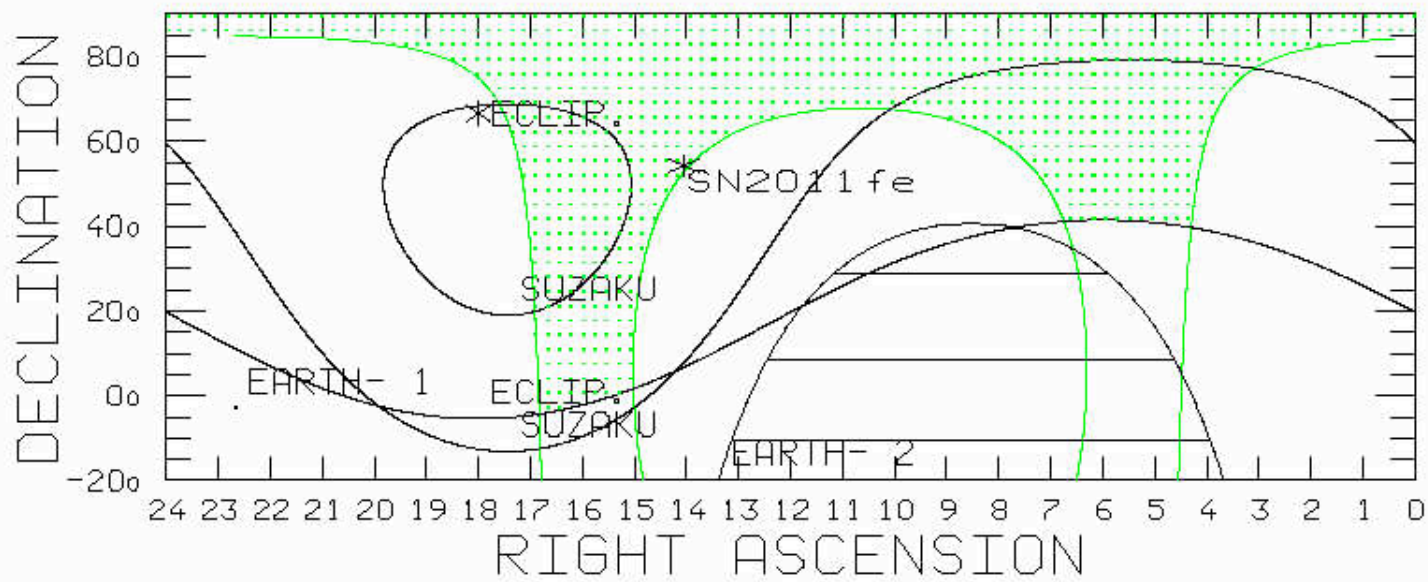

Figure 5. The large, complex error box of GRB 110823 (shaded in green). It is composed of a wide IPN annulus and an ecliptic latitude band. Parts of the localization are excluded by Earth-blocking. 


\section{Uses of the IPN Data}

The IPN localization data are public, and may be used for any project. Some of projects which they have been or are being used for, are the following. 1) Refining Fermi GBM positions by up to 4 orders of magnitude, and refining or confirming LAT positions $(1,2)$. 2) Searching for GRBs consistent with optically detected Ib/c SNe, such as 2010ah (3), 2010ay, PTF10qts, 2011dh, and 2011fe. 3) Searching for gravitational radiation using LIGO and Virgo data (4). And 4) searching for neutrino emission using AMANDA (5) \& IceCube.

Two things to keep in mind before starting any project with the localization data are first, that the database is not fully populated. There are numerous gaps, which can be filled fairly easily if and when there is a need for it. Second, very few IPN localizations are announced in GCN Circulars. Doing so would be time-consuming, and most bursts do not warrant rapid follow-up. However, data are available for them. Users should feel free to consult the first author for advice.

\section{Acknowledgments}

$\mathrm{KH}$ is grateful for IPN support under the following NASA grants: NNX07AR71G, NNX11AP96G, NNX12AD68G, and NNX12AE41G.

\section{References}

[1] W. Zheng and C. Akerlof, GBM trigger 353549464 (GRB 120316A?) : High-energy photon emission in Fermi/LAT data, GCN Circ. 13070 (2012).

[2] K. Hurley et al., IPN Triangulation of GRB 120316A, GCN Circ. 13073 (2012).

[3] A. Corsi et al., PTF 10bzf (SN 2010ah): A Broad-line Ic Supernova Discovered by the Palomar Transient Factory, ApJ 741 (2012) 76.

[4] J. Abadie et al., Search for Gravitational Wave Bursts from Six Magnetars, ApJ 734 (2012) L35.

[5] A. Achterberg et al., The Search for Muon Neutrinos from Northern Hemisphere Gamma-Ray Bursts with AMANDA, ApJ 674 (2008) 357. 